University of Wollongong

Research Online

Faculty of Informatics - Papers (Archive)

Faculty of Engineering and Information

Sciences

4-3-2007

\title{
A Study of Different Angles for the New Spread Matrix for BSOFDM in UWB Channels
}

Ibrahim S. Raad

University of Wollongong, ibrahim@uow.edu.au

Xiaojing Huang

University of Wollongong, huang@uow.edu.au

Darryn Lowe

University of Wollongong, darrynl@uow.edu.au

Follow this and additional works at: https://ro.uow.edu.au/infopapers

Part of the Physical Sciences and Mathematics Commons

\section{Recommended Citation}

Raad, Ibrahim S.; Huang, Xiaojing; and Lowe, Darryn: A Study of Different Angles for the New Spread Matrix for BSOFDM in UWB Channels 2007.

https://ro.uow.edu.au/infopapers/547

Research Online is the open access institutional repository for the University of Wollongong. For further information contact the UOW Library: research-pubs@uow.edu.au 


\title{
A Study of Different Angles for the New Spread Matrix for BSOFDM in UWB Channels
}

\author{
Abstract \\ This paper presents a study into different angles for the New spread matrix developed for BSOFDM. It \\ varies the angles for the matrix to develop different constellation schemes which are useful in \\ overcoming the frequency selective channels which are encountered in mobile communication systems. \\ Previously it has been discussed that this new matrix (the rotation matrix) has some advantages over \\ Hadamard and the rotated Hadamard matrix in certain channels. This paper presents a study of varies \\ angles with this new matrix over the UWB channels CM1 to CM4.

\section{Disciplines} \\ Physical Sciences and Mathematics

\section{Publication Details} \\ This paper was originally published as: Raad, IS, Huang, X \& Lowe, D, A Study of Different Angles for the \\ New Spread Matrix for BSOFDM in UWB Channels, Third International Conference on Wireless and Mobile \\ Communications 2007 (ICWMC '07), Guadeloupe, French Caribbean, 4-9 March 2007, 67-67. Copyright \\ IEEE 2007.
}




\title{
A Study of Different Angles for the New Spread Matrix for BSOFDM in UWB Channels
}

\author{
Ibrahim S. Raad, Xiaojing Huang and Darryn Lowe \\ School of Electrical, Computer and Telecommunications Engineering \\ University of Wollongong, N.S.W Australia \\ ibrahim@uow.edu.au
}

\begin{abstract}
This paper presents a study into different angles for the New spread matrix developed for BSOFDM. It varies the angles for the matrix to develop different constellation schemes which are useful in overcoming the frequency selective channels which are encountered in mobile communication systems. Previously it has been discussed that this new matrix (the rotation matrix) has some advantages over Hadamard and the rotated Hadamard matrix in certain channels. This paper presents a study of varies angles with this new matrix over the UWB channels $C M 1$ to $C M 4{ }^{1}$
\end{abstract}

Key Words-OFDM, Spreading Matrices, Block SpreadOFDM, UWB

\section{Introduction}

Many solutions have been presented to allow a communications system to improve its spectral efficiency of the modulation schemes by applying different schemes such as adaptive modulation based on the Bit Error Rate (BER) or the signal to noise ratio. But these systems suffer from complexity issues and the fact many still, after varies algorithms to improve the spectral efficiency, end up using BPSK or QPSK. This paper continues the study of a new method to increase the correlation between the symbols through the use of a rotation of the modulated symbols, and depending on the rotation angle, $\alpha$, a new and higher order modulation is used in the transmission of the system to increase the correlation between the transmitted symbols to improve the BER performance. This new spreading matrix [1], as with other existing matrices like the Hadamard, is used in Block Spreading OFDM and is discussed in detail in [2], [3], [4], [5] and [6]. At the receiver, the decoder used is the Maximum Likelihood decoder (ML). This is not the same as adaptive modulation as this does not retransmit the data when the new modulation scheme is created but rather has the same modulation scheme at the transmission. This paper has the following sections. Section 2 provides a description of the system

${ }^{1}$ This research is sponsored by ARC DP 0558405 used to test the New matrix and discusses the advantages and disadvantages of this New spread matrix over other existing spreading matrix. Section 3 discusses the new spreading matrix used in this study. A brief description is given of the four UWB channel models in Section 4. Section 5 gives the results achieved with the new spreading matrix and the comparisons between it and the existing spreading matrices over UWB channel models. And finally in Section 6 provides a conclusion.

\section{System Description}

Primarily this new spreading matrix is used in what has been described as Block Spread OFDM (BSOFDM), which is when the full set of subcarriers are divided into smaller blocks and using spreading matrices to spread the data across these blocks so to achieve multipath diversity across each block at the receiver [2], [3], [4] and [5]. The BSOFDM channel model is shown in Figure 1.

$$
y=C q+n
$$

The output of the receiver's FFT processor is shown in Equation 1, where $y$ is the FFT output, $q \in A^{N}$ is the vector of transmitted symbols, each drawn from an alphabet $A, C$ is a diagonal matrix of complex normal fading coefficients, and $n$ is a zero mean complex normal random vector. Equalization of the received data is done through multiplication by $C^{-1}$ and then "quantized independently on each subcarrier to form the soft or hard decision $\hat{q}$ which may be further processed if the data bits are coded" [4]. There is no loss in performance when the detection is performed independently on each carrier due to the noise being independent and identically distributed with fading been diagonal [4].

The block spreading matrices are used to introduce dependence among the subcarriers. $N$ subcarriers are split into $\frac{N}{M}$ of blocks of size $\mathrm{M}$, where $M=2$ for this example. Then each of the blocks are multiplied by a $2 \times 2$ unitary matrix $U_{2}$. The length two output vectors are interleaved using general block interleaving to ensure the symbols are statistically independent so as to encounter 


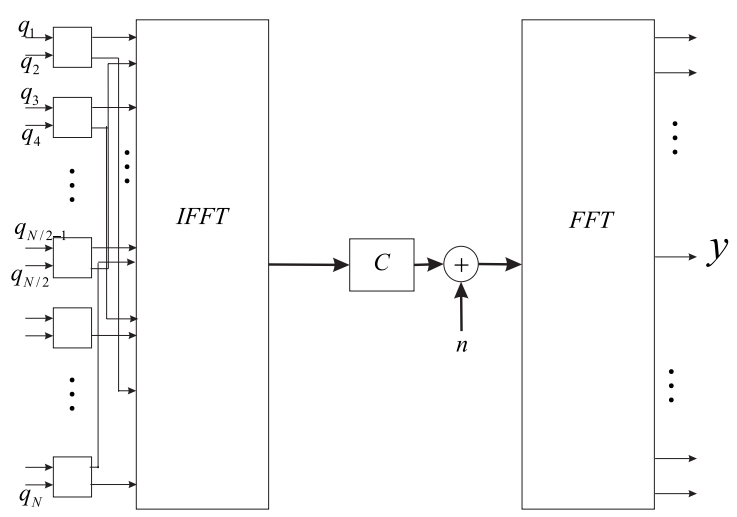

Figure 1. Block diagram representation of the BSOFDM channel for a block length of two [4].

independent fading channels. This will ensure in a dispersive frequency selective channel the data is statistically less likely to become corrupted and studies and simulations have shown this to be correct.

The transmitter's IFFT has the interleaved data passed through it and this data is sent across the frequency selective channel. The data is passed through an FFT processor at the receiver and deinterleaved before using block by block processing.

The spreading matrices are generally used to increase the correlation between the transmitted symbols after the transmission has occurred. Unlike adaptive modulation schemes where depending on the system, a higher order modulation scheme is used to retransmit the data depending on the conditions presented, this scheme utilizes spreading matrices to increase the correlation between the symbols, rather than retransmitting. This is depicted in Figure 2. So say at the transmission the system modulates the data using QPSK modulation, with spreading matrices a higher order modulation is used to increase correlation and therefore overall system performance. There are a number of matrices available and well studied, this paper continues the study of the new matrix presented in [1] concentrating on the various angles which can be used with this new matrix. Below the description of the new matrix is presented and discussed in terms of the different possible combinations achievable.

\section{New Spreading Matrix}

The following is a description of the new spread matrix for BSOFDM, where for example to make QPSK into 16QAM the choice of $\tan (\alpha)=0.5$. This achieves different modulation schemes to that of Hadamard and Rotated Hadamard.

$$
A=\left[\begin{array}{cc}
1 & \tan (\alpha) \\
\tan (\alpha) & -1
\end{array}\right]
$$

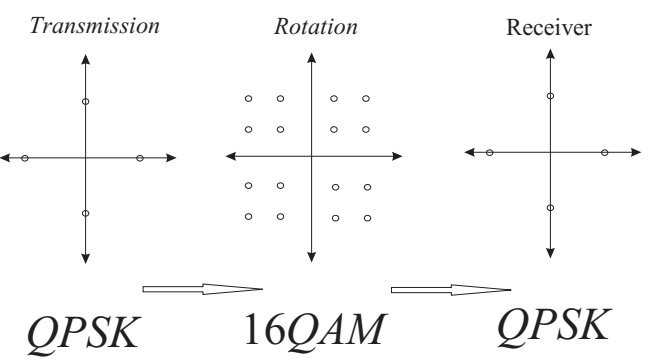

Figure 2. The process through which the transmitted modulation is converted into a higher order modulation and then returned at the receiver.

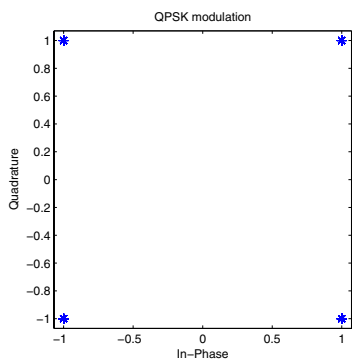

Figure 3. The QPSK constellation points.

Depending on the choice of $\alpha$, different modulation schemes are possible. Naturally, not all angles can be chosen since this would not yield a better result than the Hadamard matrix. For example an angle of $\alpha=\frac{\pi}{4}$ would result in one, which would mean that the matrix is a Hadamard matrix. Other angles which cannot be used when using QPSK are $\alpha=\pi$ and $\frac{\pi}{2}$ since the rotation of QPSK would rotate back onto it self and the new rotation would be the same as the rotated, that is QPSK. Other angles then can be used and are discussed below.

The following figures depict the varying constellation points which are achievable using the new spreading matrix. Figure 3 depicts the constellation scatter plot of QPSK which will be used for this study. Figures 4, 5, 6, 7, 8 and 9 depict the different constellation points which are achievable using the new matrix. As can be seen from Figure 9 , since $\tan \left(\frac{\pi}{4}\right)=1$, the end result is the same as the Hadamard matrix.

\section{UWB channels}

Ultra-wideband (UWB) systems occupy - by definition - a signal spectrum of more than $500 \mathrm{MHz}$ or more than $20 \%$ with regards to their centre frequency. The application of such large bandwidths enables communication systems with unique novel properties, like high-precision 


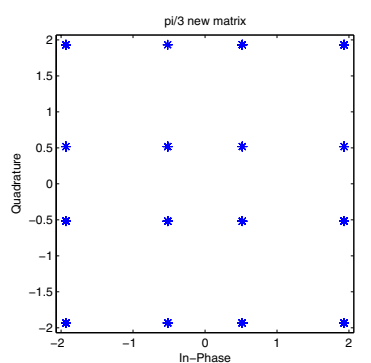

Figure 4. The new matrix for block spread OFDM with rotation $\frac{\pi}{3}$.

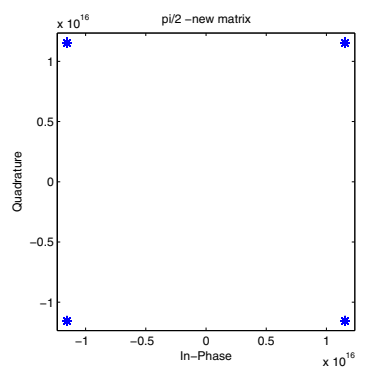

Figure 5. The new matrix for block spread OFDM with rotation $\frac{\pi}{2}$.

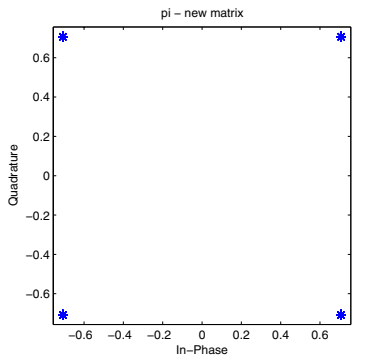

Figure 6. The new matrix for block spread OFDM with rotation $\pi$.

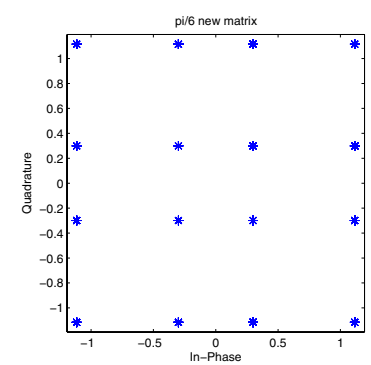

Figure 7. The new matrix for block spread OFDM with rotation $\frac{\pi}{6}$.

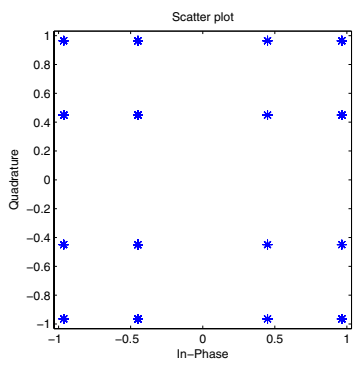

Figure 8. The new matrix for block spread OFDM with rotation $\frac{\pi}{9}$.

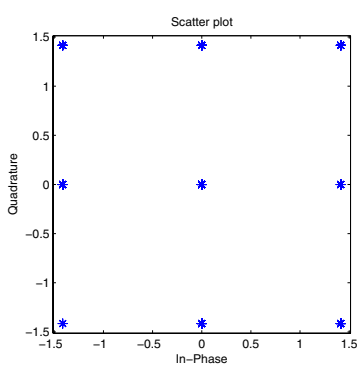

Figure 9. The new matrix for block spread OFDM with rotation $\frac{\pi}{4}$. 


\begin{tabular}{|c|c|c|c|c|}
\hline & CM1 & CM2 & CM3 & CM4 \\
\hline Channel Model & LOS & NLOS & NLOS & NLOS \\
& $0-4 m$ & $0-4 m$ & $4-10 m$ & $10 m$ \\
Mean Ex.delay(ns) & 5 & 9.9 & 15.9 & 30.1 \\
RMS Delay(ns) & 5 & 8.0 & 15.0 & 25.0 \\
No.of multi-arrival & 12.5 & 15.3 & 24.9 & 41.2 \\
within 10dB of peak & & & & \\
\hline
\end{tabular}

Table 1. UWB channels defined by IEEE [7]

indoor positioning.

Based on the Saleh-Valenzuela model for indoor multipath radio propagation channels, a set of statistically UWB channel models has been produced by the IEEE .802.15.3a task group [7]. Line of Sight (LOS) and Non-Line of Sight (NLOS) cases can be modelled using these standard channel models. The four different scenarios are summarized in table 1. Each are identified from $C M 1$ to $C M 4$. These channels are used in our study of the various angles with the new spreading matrix for BSOFDM.

\section{Results}

The results below depict the simulation results for different angles used with the new spreading matrix. The channel used for this study is UWB described in Table 1. In [1], a comparison of the new Spreading matrix was discussed and the results of a similar study is shown in Figure 10 using the UWB $C M 1$ model. As can be seen the new matrix outperforms the Hadamard matrix significantly. The Rotated Hadamard has a very similar performance to the new matrix and to the Rotated new matrix as can be seen from Figure 10. So the advantage of this new matrix is its flexibility in achieving the same matrices as that of the Hadamard and Rotated Hadamard and other combinations as well based on the rotation of the angle. This study sets out to study the angle which achieves the best result in UWB channel models.

In Figure 11, using the UWB channel model $C M 1$, it can be seen that the angle $\frac{\pi}{3}$ achieves the best performance in terms of BER in a BSOFDM system.

In Figure 12, the packet error rate of the angle $\frac{\pi}{3}$ can be seen having the best performance for the BSOFDM system over UWB channel model $C M 1$.

As can be seen in Figure 13, the performance of different angles with the new spreading matrix with the UWB channel $C M 1$, the angle $\frac{\pi}{3}$ outperforms the other angles. Angle $\frac{\pi}{6}$ has a similar performance. It is interesting to note that the angle $\frac{\pi}{4}$ has a similar performance to that of the Hadamard matrix since the $\tan \left(\frac{\pi}{4}\right)=1$ and substituting this value into the new matrix will result in a Hadamard matrix. This can be seen from the constellation point diagram seen in Figure 9. As discussed earlier, the two angles $\frac{\pi}{2}$ and $\pi$ will yield the same result as the QPSK modulation, this can be seen in Figure 13. QPSK modulation is modelled under the heading normal. The reason for this is that the two mentioned angles simply rotate the QPSK

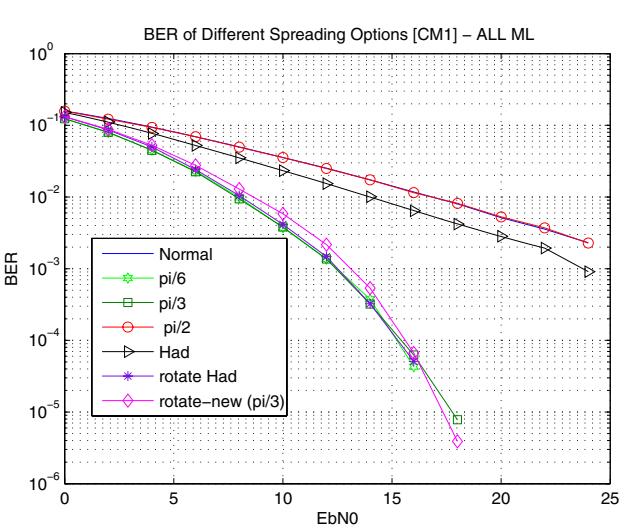

Figure 10. Comparing the new matrix with varying angles versus the Hadamard and Rotated Hadamard.

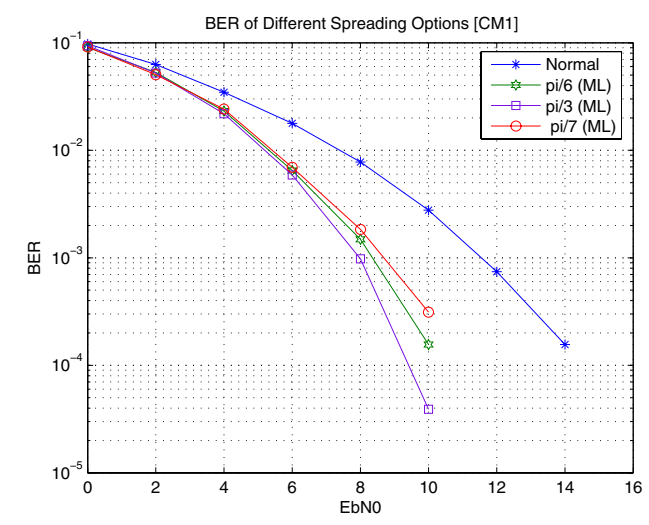

Figure 11. The new matrix with angles $\frac{\pi}{6}, \frac{\pi}{3}$ and $\frac{\pi}{7}$.

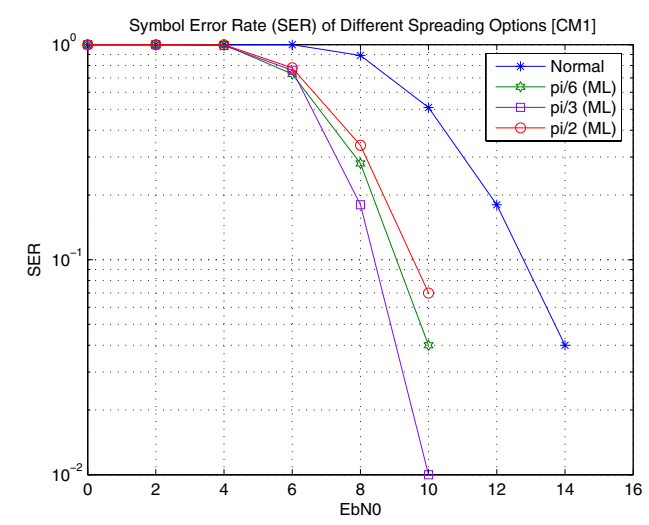

Figure 12. The new matrix with angles $\frac{\pi}{6}, \frac{\pi}{3}$ and $\frac{\pi}{7}$. 


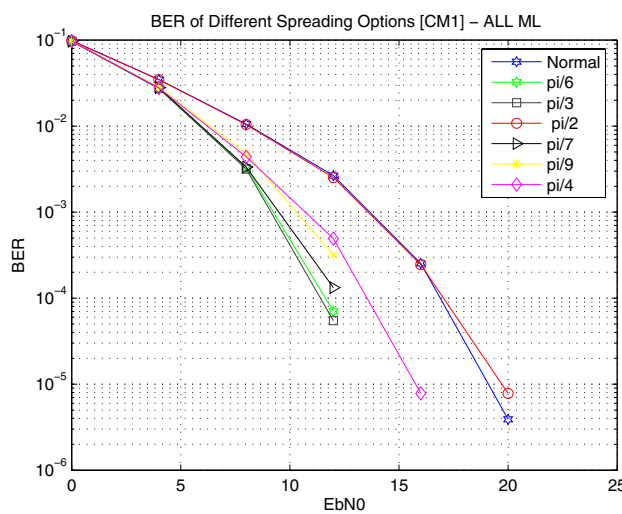

Figure 13. The new matrix with angles $\frac{\pi}{6}, \frac{\pi}{3}, \frac{\pi}{2}, \frac{\pi}{7}, \frac{\pi}{9}$ and $\frac{\pi}{4}$ using $C M 1$ channel.

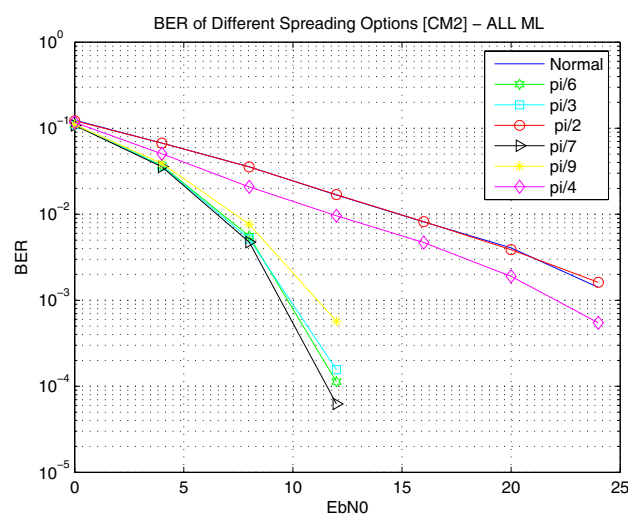

Figure 14. The new matrix with angles $\frac{\pi}{6}, \frac{\pi}{3}, \frac{\pi}{2}, \frac{\pi}{7}, \frac{\pi}{9}$ and $\frac{\pi}{4}$ using $C M 2$ channel.

modulation back onto it self. This can be seen from the constellation points presented above in Figures 5 and 6.

For the UWB channel model $C M 2$, it can be seen in Figure 14 that the angle $\frac{\pi}{7}$ out performs all the others, again we see that Hadamard is outperformed significantly.

For both the UWB channel models $C M 3$ and $C M 4$ the angles $\frac{\pi}{3}$ and $\frac{\pi}{7}$ again outperform the other angles shown, this can be seen in Figures 15 and 16. Again it can be seen that the Hadamard matrix (also the angle $\frac{\pi}{4}$ ) are outperformed by a significant margin. It can be seen that the angle $\frac{\pi}{6}$ has a similar performance to that of the angles $\frac{\pi}{3}$ and $\frac{\pi}{7}$. The angle $\frac{\pi}{9}$ does not perform as well, although it does out perform the Hadamard. This is due to the separation of the constellation points for each scheme. The more equal the distance apart is from each constellation point, the better the performance will be. Future work will concentrate on finding the optimum angle for this matrix to achieve that goal.

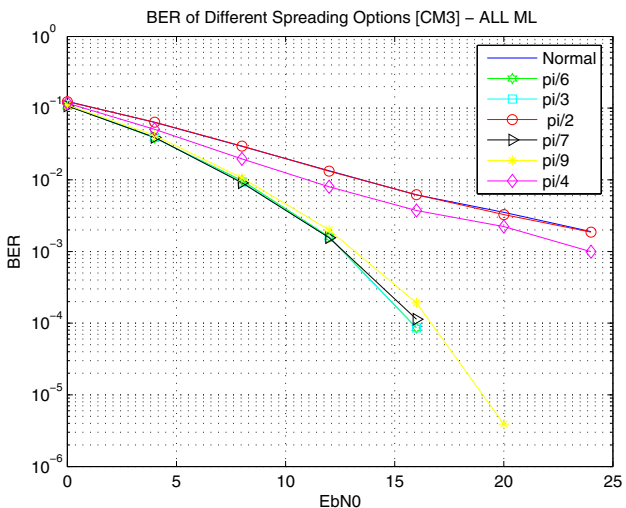

Figure 15. The new matrix with angles $\frac{\pi}{6}, \frac{\pi}{3}, \frac{\pi}{2}, \frac{\pi}{7}, \frac{\pi}{9}$ and $\frac{\pi}{4}$ using $C M 3$ channel.

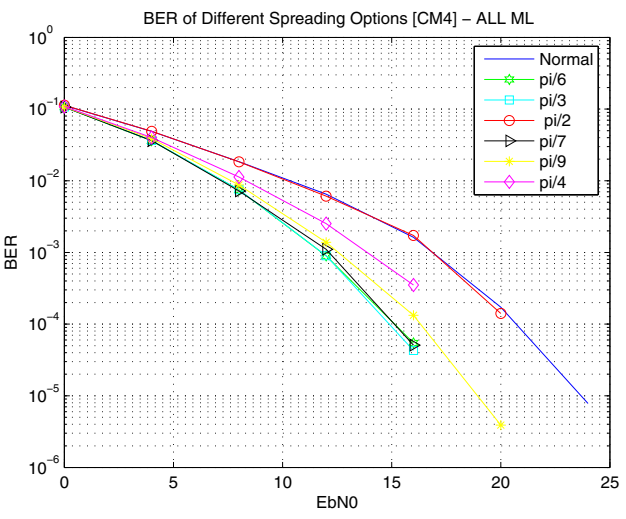

Figure 16. The new matrix with angles $\frac{\pi}{6}, \frac{\pi}{3}, \frac{\pi}{2}, \frac{\pi}{7}, \frac{\pi}{9}$ and $\frac{\pi}{4}$ using $C M 4$ channel. 


\section{Conclusion}

In conclusion this paper gave a study for varying angles which can be used for the new spreading matrix proposed in [1] for BSOFDM system over the four models proposed for the IEEE.15.3a task group. This paper studies what the different constellation points are and what, if any, improvement take place for the common modulation schemes. It can be said that the limitations of such a spread matrix is the angles which reproduce the same constellation scheme as that of the modulation scheme used. In other words when using for example a QPSK modulation scheme, the use of the angle for example $\frac{\pi}{2}$, would simply rotate the modulation on it self as has been shown and end up using the same modulation scheme again.

The advantages can be noted is its flexibility in determining different structures of matrices and the simple angle rotation allows an improvement to take place over more traditional spreading matrices. At the same time, this matrix can reproduce existing matrices depending on the angle used, for example $\frac{\pi}{4}$ would result in the Hadamard matrix.

It can be stated at the end of this simple study, that the angles which perform the best over UWB channel models are the angles $\frac{\pi}{3}, \frac{\pi}{6}$ and $\frac{\pi}{7}$. Angles $\frac{\pi}{4}, \frac{\pi}{2}$ and $\pi$ do not achieve the same performance as those listed above.

Future work on this matrix will include finding the theoretical optimum angle to achieve the best result for this new matrix.

\section{References}

[1] Ibrahim Raad, Xiaojing Huang and Raad Raad, "A New Spreading Matrix for Block Spread OFDM" 10th IEEE International Conference on Communication Systems 2006 (IEEE ICCS'06), Singapore, 31 October - 3 November 2006.

[2] Michael L. McCloud, "Coded block OFDM for the frequency selective fading channel", Signals, Systems and Computers, Conference Record of the Thirty-Eighth Asilomar Conference on, volume 1, 2004, 329-333, November.

[3] Michael L. McCloud, "Analysis and Design of Short Block OFDM Spreading Matrices for Use on Multipath Fading Channels", Communications, IEEE Transactions on, volume 53, 4, 2005, 656-66, April.

[4] Michael L. McCloud, "Optimal Binary Spreading for Block OFDM on Multipath Fading Channels", WCNC / IEEE Communications Society, volume 2, 2004, 965-970, March.

[5] Ibrahim S. Raad, Xiaojing Huang, "Exploiting Time diversity to improve Block Spread OFDM", First IEEE International Conference on Wireless Broadband and Ultra Wideband Communications, Aus Wireless 2006, Sydney, 13 - 16 March.
[6] Ibrahim S. Raad and Xiaojing Huang, "Exploiting Time Diversity to Improve Block Spread OFDM in a Multipath Environment" The Second International Conference on Information \& Communication Technologies - From Theory to Applications, IEEE ICTTA'06, Damascus, Syria, 24 - 28 April 2006.

[7] Darryn W. Lowe, "Real - time FPGA Realization of an UWB transceiver physical layer" Thesis for Masters of Engineering Research at the University of Wollongong, The School of Electrical, Computer and Telecommunications Engineering, 2005. 